\section{Las enfermedades emergentes y reemergentes: un problema de salud en las Américas ${ }^{1}$}

\author{
Guillermo Mesa Ridel,,2 Iraida \\ Rodríguez Luis ${ }^{2}$ y Julio Teja²
}

Palabras clave: enfermedades emergentes y reemergentes, Américas.

\footnotetext{
Basado en la relatoría de la sesión "Las enfermedades emergentes y reemergentes" del VI Congreso Internacional Sobre Desastres, Ciudad de La Habana, Cuba, 25 al 28 de noviembre de 2003 (en preparación).

2 Centro Latinoamericano de Medicina de Desastres, Ciudad de La Habana, Cuba. La correspondencia debe dirigirse a Guillermo Mesa, Centro Latinoamericano de Medicina de Desastres, Calle 18 No. 710, Playa, Ciudad de La Habana, Cuba. Correo electrónico: mesa@clamed. sld.cu

3 Enfermedades que se observan en el ser humano por primera vez.

4 Enfermedades que habían sido controladas pero que están volviendo a aparecer con ímpetu en la población.
}

Las enfermedades infecciosas constituyen la primera causa de muerte en el mundo, tanto en adultos como en niños. Más de 13 millones de personas mueren anualmente por enfermedades infecciosas emergentes $^{3}$ y reemergentes, ${ }^{4}$ tales como la malaria, la tuberculosis, el síndrome de la inmunodeficiencia adquirida (sida), la fiebre hemorrágica producida por el virus Ébola, el síndrome respiratorio agudo grave (SARS), la infección por el virus del Nilo occidental y el dengue (1). Solo tres de estas infecciones (el sida, la tuberculosis y la malaria) cobraron 5,7 millones de vidas durante el año 2001, la mayor parte de ellas en países en desarrollo (1). A pesar de que la infección por el virus de la inmunodeficiencia humana (VIH) y el síndrome de inmunodeficiencia adquirida (sida) han estado presentes desde hace más de 20 años, aún se incluyen en la mayoría de los análisis nacionales y regionales relacionados con las enfermedades emergentes debido a su elevado costo social y sanitario.

En la Región de las Américas, las enfermedades infecciosas emergentes y reemergentes que tuvieron una mayor repercusión sobre la salud de la población por su incidencia y por el número de muertes ocasionadas durante el quinquenio de 1999-2003 fueron: la malaria, la fiebre amarilla, el dengue hemorrágico, el sida, el carbunco y el SARS, así como la infección por hantavirus y por el virus del Nilo occidental $(2,3)$. Algunas de estas enfermedades, como el SARS, presentan una distribución geográfica focal, mientras que otras, como el dengue, se dispersan ampliamente y se han convertido en un problema mundial (2).

La aparición de enfermedades emergentes y reemergentes se asocia con factores de diversa índole. Si bien el origen y la evolución de los agentes etiológicos han sido suficientemente documentados en la mayoría de los casos, la aparición de estas enfermedades depende de una compleja interacción de distintos factores, tanto biológicos - la variabilidad y adaptabilidad genética de los microorganismos causantes - como sociales - el auge del comercio internacional y de los movimientos poblacionales por razones turísticas o migratorias, el hacinamiento, la inadecuada manipulación de los alimentos y el analfabetismo, entre otros- y económicos - la ausencia de servicios básicos de saneamiento ambiental, la desnutrición, la falta de agua y de sistemas de alcantarillado (3). Todos estos factores crean, además, las condiciones propicias para la propagación de estas infecciones y facilitan la circulación de los agentes 
patógenos y la transmisión de las infecciones y epidemias entre los diversos países y regiones, situación agravada por las ineficiencias de los sistemas de salud pública, entre ellas una vigilancia epidemiológica deficiente, un número insuficiente de laboratorios de salud pública para la detección y el diagnóstico de las enfermedades, y mecanismos de prevención y de control inadecuados.

La experiencia acumulada demuestra que una gran parte de las muertes que se producen debido a las enfermedades infecciosas emergentes y reemergentes podrían evitarse mediante la elaboración y puesta en marcha de estrategias preventivas y terapéuticas eficaces (4).

Para poder evitar o mitigar los graves efectos de estas epidemias, los países deberán darle un lugar prioritario en la agenda nacional a la vigilancia de las enfermedades emergentes y reemergentes y poner en marcha un conjunto de medidas para combatirlas. Entre las medidas destinadas a prevenir y mitigar las epidemias deberán figurar las siguientes: a) establecer una estrategia basada en mecanismos de alerta temprana y de respuesta rápida que cuente con recursos humanos, laboratorios, redes de comunicación entre los laboratorios y servicios de salud, y que esté respaldada por una prioridad financiera y política adecuada; b) fortalecer la capacidad nacional de adoptar estrategias para la prevención y el control de las enfermedades emergentes y reemergentes; c) promover la investigación aplicada al diagnóstico rápido y tratamiento de las enfermedades emergentes y reemergentes y a la prevención de sus factores de riesgo; d) fortalecer la red de comunicaciones entre los laboratorios y servicios de salud para mejorar la vigilancia de los agentes infecciosos; e) mantener la vigilancia sistemática de los vectores y reservorios de las enfermedades emergentes y reemergentes; f) sistematizar la vigilancia de los factores de riesgo y elementos ambientales y climáticos que favorecen la aparición de epidemias; y g) crear una estructura para la vigilancia integral de los agentes causales y factores de riesgo que permita analizar la información de forma rápida y eficaz a fin de tomar decisiones oportunas con la mayor celeridad posible.

La prevención de epidemias, epizootias y plagas, así como la toma de medidas adecuadas para mitigarlas, no es solo responsabilidad de los organismos profesionales que normalmente se encargan de estas actividades en los diferentes países. Por tratarse de situaciones de desastre es necesario que la participación sea multisectorial, conforme a la magnitud del impacto de estas enfermedades sobre la población y la economía, especialmente en los países pobres.

En resumen, el elemento básico de cualquier estrategia de lucha contra las enfermedades emer- gentes y reemergentes y las epidemias que estas pueden causar es el establecimiento de un sistema de vigilancia orientado a detectar la presencia de esas enfermedades a tiempo para tomar medidas de control adecuadas.

El tema de las enfermedades emergentes y reemergentes se debatió extensamente en el VI Congreso Internacional sobre Desastres y en el II Seminario Internacional de Administración de Riesgos, que se celebraron conjuntamente en La Habana del 25 al 28 de noviembre de 2003. Los expertos que asistieron a estas reuniones, convencidos de la necesidad de ampliar el enfoque multisectorial en los debates acerca de la lucha contra las enfermedades emergentes y reemergentes, hicieron un llamado a la creación de mecanismos eficaces y eficientes para ampliar la colaboración en este sentido con la ayuda de las organizaciones internacionales interesadas en el tema. Los expertos también coincidieron en la necesidad de convocar, con el auspicio de organizaciones internacionales que intervienen en situaciones catastróficas, un encuentro entre especialistas para debatir con mayor amplitud y profundidad las experiencias nacionales y los aspectos metodológicos y técnicos del asunto, especialmente los relacionados con los estudios sobre los factores de riesgo de desastres.

La lucha contra el flagelo de las enfermedades emergentes y reemergentes en la Región debe responder al planteamiento de Gro Harlem Brundtland, antigua Directora General de la Organización Mundial de la Salud: "These are dangerous times for the well-being of the world. In many regions, some of the most formidable enemies of health are joining forces with the allies of poverty to impose a double burden of disease, disability and premature death on many millions of people. It is time for us to close ranks against this growing threat" (5).

\section{SYNOPSIS}

\section{Emerging and reemerging diseases: a health problem in the Americas}

In the Region of the Americas the emerging and reemerging infectious diseases that had the greatest impact on health, in terms of their incidence and the number of deaths that they caused during the five-year period of 1999-2003, were: malaria, yellow fever, dengue hemorrhagic fever, AIDS, anthrax, and $S A R S$, as well as infection by hantavirus and by West Nile virus. The appearance of epidemics of emerging and reemerging diseases is related to biological, social, and economic factors. Growth in international trade, the movement of large numbers of people across national borders, the variability and genetic adaptability of the causative mi- 
croorganisms, and inefficiencies in public health systems help to spread infections and epidemics. To avoid or reduce the serious effects of these epidemics, countries should give priority in their national agendas to surveillance of emerging and reemerging diseases and should implement a set of measures to combat the diseases. The most important of these measures is to develop a strategy that is based on early warning and rapid response mechanisms, with personnel and laboratories as well as communications networks that link laboratories with health service providers. This strategy should be backed by priority funding and adequate policies.

\section{REFERENCIAS}

1. World Health Organization. Scaling up the response to infectious diseases. Geneva: WHO; 2001. Hallado en: http:// www.who.int/infectious-disease-report/ 2002. Acceso el 4 de marzo de 2004.

2. Organización Panamericana de la Salud. Enfermedades infecciosas nuevas, emergentes y reemergentes. Bol Epidemiol. 1995;16(3):1-7.
3. Organización Panamericana de la Salud. Enfermedades infecciosas emergentes y reemergentes. En: La salud en las Américas. Washington, D.C.: OPS; 2002. (Publicación Científico y Técnica No. 587).

4. Rojas Ochoa F. Situación, sistema y recursos humanos en salud para el desarrollo en Cuba Modelo de lucha contra epidemias. Rev Cubana Salud Publica. 2003;29:
157-69. Hallado en: http://bvs.sld.cu/ revistas/spu/vol29_2_03/spu11203.htm. Acceso el 8 de marzo de 2004.

5. World Health Organization. The World Health Report 2002. Geneva: WHO; 2002. Hallado en: http://www.who.int/whr/ 2002/en/. Acceso el 4 de marzo de 2004.

\section{Fallece el Dr. Myron E. Wegman, distinguido salubrista de fama internacional}

La noticia del fallecimiento del Dr. Myron E. Wegman el 14 de abril entristece a la comunidad científica, que lo recuerda como un activo defensor de la salud infantil en el mundo y un salubrista de primera talla. El Dr. Wegman, originario de Brooklyn, Nueva York, falleció a los 95 años de edad después de siete decenios de trabajo en pediatría clínica y en el campo de la salud pública. Durante 14 años (1960-1974) fue decano de la Facultad de Salud Pública de la Universidad de Michigan, Estados Unidos de América, cuyas actividades de investigación en el ámbito internacional se vieron fortalecidas bajo su dirección. Enseñó salud internacional y pediatría en la facultad de medicina de esa institución, incluso hasta después de jubilarse en 1978, cuando empezó a dedicarse más de lleno a actividades de salud pública en los niveles local, estatal, nacional e internacional. El Dr. Wegman fue funcionario de la Organización Panamericana de la Salud durante ocho años y los tres últimos fungió como su Secretario General (1957-1960).

El Dr. Wegman también ocupó cargos académicos importantes en otras universidades, entre ellas las de Yale, Cornell, Columbia y Johns Hopkins, y fue jefe del departamento de pediatría en la Universidad del Estado de Luisiana. Recibió numerosas condecoraciones a lo largo de los años, destacándose entre ellas el Premio Bronfman que le otorgó la Asociación Estadounidense de Salud Pública en 1967 y el Premio Sedgwick, ambos por su extraordinaria labor en el campo de la salud pública.

Su obra científica comprende más de 200 artículos, algunos dedicados a la pediatría y otros a temas vinculados con las políticas y decisiones administrativas en el ámbito de la salud pública. De 1949 a 1997 fue redactor de un informe de estadísticas vitales que se publicaba en cada número de diciembre de la revista $P e-$ diatrics, tarea que abandonó con renuencia debido al peso de los años.

La Revista Panamericana de Salud Pública le rinde este humilde homenaje a la memoria del Dr. Wegman y le extiende su más sentido pésame a la familia que lo sobrevive. 